\title{
Nuances of transarterial and transvenous embolization of a large tentorial arteriovenous fistula
}

\author{
Lorenzo Rinaldo, MD, PhD, ${ }^{1}$ Waleed Brinjikji, MD,1,2 and Leonardo Rangel-Castilla, MD1,2 \\ Departments of ${ }^{1}$ Neurosurgery and ${ }^{2}$ Radiology, Mayo Clinic, Rochester, Minnesota
}

An 80-year-old female presented with a long history of severe pulsatile tinnitus, vertigo, and decreased hearing. She was found to have a large right-sided tentorial arteriovenous fistula (AVF) with enlarged deep draining veins, including the vein of Rosenthal. The patient underwent Onyx embolization of the fistula via a combined transarterial and transvenous approach resulting in complete obliteration of the fistula. Her symptoms improved immediately after the procedure and at 6-months' follow-up she was clinically asymptomatic with no evidence of residual fistula on neuroimaging. Transvenous embolization of AVF is at times necessary when transarterial access is not possible.

The video can be found here: https://youtu.be/uOMHY7eaOoQ.

KEYWORDS arteriovenous fistula; endovascular procedures; tentorium cerebelli; Onyx; video 